HUTP-92/A026

\title{
Two Stage Phase Transition in Two Higgs Models
}

\author{
David Land* \\ Eric D. Carlson* \\ Lyman Laboratory of Physics \\ Harvard University \\ Cambridge, MA 02138
}

\begin{abstract}
We present a mechanism in which models with two Higgs fields can undergo a two stage phase transition as the temperature falls. The first stage is a conventional second order (or weakly first order) transition in which the symmetry is broken. Shortly thereafter follows a first order transition with barrier penetration, bubble production and loss of thermal equilibrium. For Higgs potentials with CP violation, we show that the second stage of the transition has all the features required for weak scale baryogenesis.
\end{abstract}

$5 / 92$

* This research was supported by the National Science Foundation under Grant \#PHY-8714654 and by the Texas National Research Laboratory Commission under Grant \#RGFY9106 


\section{Introduction}

The $\mathrm{SU}(2)_{L} \times \mathrm{U}(1)_{\mathrm{Y}}$ symmetry of the standard model is broken in the early universe as the temperature falls below the weak scale. The order parameter for the phase transition is the real component of the neutral Higgs field, which acquires a non-zero vacuum expectation value below the transition temperature. The precise details of the transition are still the subject of controversy, though the consensus seems to be that it is weakly first order for Higgs masses greater than the experimental lower bound [1 3 ]. That is, at the transition, the Higgs vacuum expectation value (VEV) makes a jump from zero to some very small fraction of its eventual zero temperature value.

Two Higgs models have also been investigated recently [4,5], though the whole range of parameter space has not been fully explored. Previous work has concentrated on first finding the zero temperature absolute minimum of the effective potential and then, in much the same way as has been done for the single Higgs model, studying the evolution of this one minimum from high to low temperatures. For certain combinations of parameters, one loop effective potential calculations suggest that the transition can be first order [6]

In this paper, we make use of the large number of parameters in a two Higgs model, and construct a potential in which the absolute minimum just after the phase transition bears no relation to the absolute minimum at zero temperature. At the transition point, the VEV is lured the "wrong" way, into a minimum which is only an absolute minimum for a short time close to the critical temperature. As the temperature continues to fall, it soon becomes a false vacuum, because a second minimum begins to appear whose depth quickly overtakes that of the first. At this point a tunneling process will occur with production of bubble walls and loss of thermal equilibrium, thus producing the main characteristics of a first order transition. As we shall see, it is not difficult to ensure that the discontinuity in the VEV is rather large. In fig. 1, these various stages of the transition are indicated.

The electroweak phase transition, strictly speaking, is second order (or at best weakly first order), and the first order phase transition occurs somewhat later. However, the jump in the VEV during the second stage can be large and thus this mechanism could be useful in generating baryons at the electroweak scale according to the scenarios recently proposed by Cohen, Kaplan and Nelson in [7]:8]. They suggest that top quarks, reflected from a bubble wall in a CP violating manner, produce a hypercharge asymmetry which is converted into a baryon asymmetry in regions of false vacuum where baryon violation is rapid. This asymmetry is swept into the true vacuum when the bubble wall passes and, 
in order that it be preserved, sphaleron processes in this region must be highly suppressed by a large scalar VEV.

In section 2, we present the simplest example of the two stage transition in a theory with just two scalar fields. In section 3, we apply the same ideas to the more complicated example of the standard model with two Higgs doublets. In section 4, we show that CP violation can be introduced into the potential whilst retaining the other important features and thus that it is suitable for weak scale baryogenesis.

\section{The Simplest Example}

Let us consider a theory of two real scalar fields $\phi_{1}$ and $\phi_{2}$ with two discrete symmetries $\phi_{1} \rightarrow-\phi_{1}$ and $\phi_{2} \rightarrow-\phi_{2}$. We will think of $\phi_{1}$ as the standard Higgs, and $\phi_{2}$ as an additional Higgs. The Lagrangian is constrained to the form:

$$
\mathcal{L}=\frac{1}{2} \partial^{\mu} \phi_{1} \partial_{\mu} \phi_{1}+\frac{1}{2} \partial^{\mu} \phi_{2} \partial_{\mu} \phi_{2}-V\left(\phi_{1}, \phi_{2}\right)
$$

where

$$
V\left(\phi_{1}, \phi_{2}\right)=k_{1} \phi_{1}^{4}+k_{2} \phi_{2}^{4}+k_{3} \phi_{1}^{2} \phi_{2}^{2}-2 \mu_{1}^{2} \phi_{1}^{2}-2 \mu_{2}^{2} \phi_{2}^{2}
$$

At zero temperature we will arrange the VEV to lie purely in the $\phi_{1}$ direction and to have a given value, in which case $\phi_{1}^{2}=\mu_{1}^{2} / k_{1}=(246 \mathrm{GeV})^{2}$ for instance, and the value of the potential here is $V_{1}=-\mu_{1}^{4} / k_{1}$. We can also arrange for there to be a second minimum, purely in the $\phi_{2}$ direction at $\phi_{2}=\mu_{2}^{2} / k_{2}$ with $V_{2}=-\mu_{2}^{4} / k_{2}$, which, if it is not to be the absolute minimum, must satisfy $\left|V_{2}\right|<\left|V_{1}\right|$. From the second derivatives of the potential at the absolute minimum it is easy to read off the zero temperature mass spectrum : $m_{1}^{2}=8 \mu_{1}^{2} ; m_{2}^{2}=2\left(k_{3} / k_{1}\right) \mu_{1}^{2}-4 \mu_{2}^{2}$. Anticipating the generalization to the real world, we suppose that there are some "experimental" constraints on these masses, let's say, that $m_{1}, m_{2}>50 \mathrm{GeV}$.

Next, we wish to make sure that the minimum which appears first during the transition is the "wrong" one, i.e., the one in the $\phi_{2}$ direction. To do this we must recompute the positions of the minima, this time as a function of temperature. To see the effect that we have in mind, it will not be necessary to go beyond leading order in high temperature corrections and for this reason we use a simple method, put forward by Linde [1], to calculate the locations of the minima. We compute thermally averaged equations of motion and assume that the particle masses are sufficiently light in comparison to the temperature 
$T$ that we can use the result $\left\langle\phi^{2}\right\rangle=T^{2} / 12$ as a good estimate of the fluctuations in the scalar fields. In this way we obtain the following pair of equations:

$$
\begin{aligned}
& \left\langle\frac{\partial V}{\partial \phi_{1}}\right\rangle=0=4 k_{1} \phi_{1}^{3}+\phi_{1}\left[\alpha_{1} T^{2}+2 k_{3} \phi_{2}^{2}-4 \mu_{1}^{2}\right] ; \\
& \left\langle\frac{\partial V}{\partial \phi_{2}}\right\rangle=0=4 k_{2} \phi_{2}^{3}+\phi_{2}\left[\alpha_{2} T^{2}+2 k_{3} \phi_{1}^{2}-4 \mu_{2}^{2}\right] ;
\end{aligned}
$$

where $\alpha_{1}=k_{1}+k_{3} / 6$ and $\alpha_{2}=k_{2}+k_{3} / 6$. The non-zero $\phi_{1}$ and $\phi_{2}$ minima appear when the temperature drops below

$$
T_{c_{1}}{ }^{2}=\frac{4 \mu_{1}^{2}}{\alpha_{1}} \quad \text { and } \quad T_{c_{2}}{ }^{2}=\frac{4 \mu_{2}^{2}}{\alpha_{2}},
$$

respectively. In order that the VEV occur first in the $\phi_{2}$ direction, we must make sure that $T_{c_{1}}<T_{c_{2}}$, even though we will end up arranging them to be not too different. Equations (2.3) can have a third solution in which both $\phi_{1}$ and $\phi_{2}$ are non-zero. In the case of interest to us, however, this will be a saddle point rather than a minimum of the effective potential.

Below these critical temperatures in this first stage of the transition, then, the minima in the effective potential lie at:

$$
\phi_{1}^{2}=\frac{4 \mu_{1}^{2}-\alpha_{1} T^{2}}{4 k_{1}} \quad, \quad \phi_{2}=0
$$

and

$$
\phi_{1}=0 \quad, \quad \phi_{2}^{2}=\frac{4 \mu_{2}^{2}-\alpha_{2} T^{2}}{4 k_{2}} .
$$

As they develop, at some temperature $T_{t}$, the depths of the two minima become equal and, below this temperature, (2.5) rather than (2.6) gives the location of the true vacuum. We assume here that bubble nucleation and conversion to the low-temperature vacuum region occurs shortly thereafter. $T_{t}$, thus, signals the onset of false vacuum decay and the second stage of the transition. The tunneling temperature can be calculated by substituting equations (2.5) and (2.6) into the temperature corrected version of (2.2) which is:

$$
V\left(\phi_{1}, \phi_{2}, T\right)=V\left(\phi_{1}, \phi_{2}\right)+T^{2} / 2\left[\alpha_{1} \phi_{1}^{2}+\alpha_{2} \phi_{2}^{2}\right]
$$

The values of the potential at the two minima are always given by $V_{1}=-k_{1} \phi_{1}^{4}$ and $V_{2}=-k_{2} \phi_{2}^{4}$ respectively, and so, if we want a large jump in the size of the VEV during the jump, we should pick the ratio $k_{2} / k_{1}$ to be large, since, when $V_{1}=V_{2}$, the ratio of the VEV's goes as the fourth root of this quantity. This ratio is restricted, however, by the 
requirement that the mass of the physical Higgs (coming from $k_{1}$ ) be not too small, and the desire to avoid extremely non-perturbative couplings. The tunneling temperature is given by:

$$
T_{t}^{2}=\frac{4\left(k_{2}^{1 / 2} \mu_{1}^{2}-k_{1}^{1 / 2} \mu_{2}^{2}\right)}{\left(k_{2}^{1 / 2} \alpha_{1}-k_{1}^{1 / 2} \alpha_{2}\right)} .
$$

It is not hard to find $k$ 's and $\mu$ 's which satisfy the three requirements of large $k_{2} / k_{1}$, $T_{c_{1}}<T_{c_{2}}$, and $\left|V_{1}\right|>\left|V_{2}\right|$ at zero temperature. For instance, suppose we choose $k_{1}=0.01$, then $\mu_{1}=25 \mathrm{GeV}$ to give a Higgs mass of $m_{1}=70 \mathrm{GeV}$. To maximize $k_{2} / k_{1}$, we choose $k_{2}$ at the edge of the perturbative regime, say $k_{2}=0.8$. A large value for $k_{3}$, such as $k_{3}=0.6$, will help us satisfy the temperature constraint. The value of $\mu_{2}$ is then constrained by the temperature and true minimum constraints; a value of $\mu_{2}=72 \mathrm{GeV}$ will suffice. The critical temperatures work out to $T_{c_{1}}=151 \mathrm{GeV}$ and $T_{c_{2}}=152 \mathrm{GeV}$, and the mass of the second scalar is $m_{2}=233 \mathrm{GeV}$. The jump takes place at $T_{t}=139 \mathrm{GeV}$, at which point the VEV changes discontinuously from $32 \mathrm{GeV}$ in the $\phi_{2}$ direction to $97 \mathrm{GeV}$ in the $\phi_{1}$ direction.

\section{The Two Higgs Doublet Standard Model}

We would now like to consider a more realistic possibility, where we include Higgs doublets $\Phi_{1}, \Phi_{2}$ instead of singlets, and we will include fermion couplings as well. The symmetries $\phi_{i} \rightarrow-\phi_{i}$ will be promoted to $\Phi_{i} \rightarrow-\Phi_{i}$, and we will allow such terms to be

softly broken. We furthermore assume that only one of these two doublets (specifically $\Phi_{1}$ ) is responsible for fermion masses, or at least, for the top quark mass; such an assumption is usually necessary to avoid flavor changing neutral currents. The potential with these constraints is conventionally written [7-9]:

$$
\begin{aligned}
V\left(\Phi_{1}, \Phi_{2}\right)= & \lambda_{1}\left(\Phi_{1}^{\dagger} \Phi_{1}-v_{1}^{2}\right)^{2}+\lambda_{2}\left(\Phi_{2}^{\dagger} \Phi_{2}-v_{2}^{2}\right)^{2} \\
& +\lambda_{3}\left[\left(\Phi_{1}^{\dagger} \Phi_{1}-v_{1}^{2}\right)+\left(\Phi_{2}^{\dagger} \Phi_{2}-v_{2}^{2}\right)\right]^{2} \\
& +\lambda_{4}\left[\left(\Phi_{1}^{\dagger} \Phi_{1}\right)\left(\Phi_{2}^{\dagger} \Phi_{2}\right)-\left(\Phi_{1}^{\dagger} \Phi_{2}\right)\left(\Phi_{2}^{\dagger} \Phi_{1}\right)\right] \\
& +\lambda_{5}\left(\operatorname{Re}\left(\Phi_{1}^{\dagger} \Phi_{2}\right)-v_{1} v_{2} \cos \xi\right)^{2} \\
& +\lambda_{6}\left(\operatorname{Im}\left(\Phi_{1}^{\dagger} \Phi_{2}\right)-v_{1} v_{2} \sin \xi\right)^{2} .
\end{aligned}
$$


In this form, one can easily be blind toward the existence of minima other than $\Phi_{1}^{0}=v_{1}$; $\Phi_{2}^{0}=v_{2} e^{i \xi}$ (because some of the coefficients above may be negative) and, for this reason, we find it more convenient to rewrite the potential as:

$$
\begin{aligned}
V\left(\Phi_{1}, \Phi_{2}\right)=\quad & 4\left\{k_{1}\left(\Phi_{1}^{\dagger} \Phi_{1}\right)^{2}+k_{2}\left(\Phi_{2}^{\dagger} \Phi_{2}\right)^{2}+k_{3}\left(\Phi_{1}^{\dagger} \Phi_{1}\right)\left(\Phi_{2}^{\dagger} \Phi_{2}\right)\right. \\
& +k_{4}\left[\left(\Phi_{1}^{\dagger} \Phi_{1}\right)\left(\Phi_{2}^{\dagger} \Phi_{2}\right)-\left(\Phi_{1}^{\dagger} \Phi_{2}\right)\left(\Phi_{2}^{\dagger} \Phi_{1}\right)\right] \\
& -\frac{1}{2}\left[k_{5}\left(\Phi_{1}^{\dagger} \Phi_{2}\right)^{2}+k_{5}^{*}\left(\Phi_{2}^{\dagger} \Phi_{1}\right)\right]^{2} \\
& \left.-\mu_{1}^{2} \Phi_{1}^{\dagger} \Phi_{1}-\mu_{2}^{2} \Phi_{2}^{\dagger} \Phi_{2}-\mu_{3}^{2} \operatorname{Re}\left(\Phi_{1}^{\dagger} \Phi_{2}\right)-\mu_{4}^{2} \operatorname{Im}\left(\Phi_{1}^{\dagger} \Phi_{2}\right)\right\}
\end{aligned}
$$

The presence of CP violation is signalled by the presence of $\mu_{4}^{2}$ and the phase of $k_{5}$. We can redefine the phase of one of the scalar fields to eliminate, for example, the phase of $k_{5}$; we will assume from now on that $k_{5}$ is real and positive (a similar phase choice was used to eliminate one term in (3.1)). The factor of 4 is present so that when we make the substitution:

$$
\Phi_{1}=\frac{1}{\sqrt{2}}\left(\begin{array}{c}
\chi_{1}+i \eta_{1} \\
\phi_{1}+i \psi_{1}
\end{array}\right) \quad ; \quad \Phi_{2}=\frac{1}{\sqrt{2}}\left(\begin{array}{c}
\chi_{2}+i \eta_{2} \\
\phi_{2}+i \psi_{2}
\end{array}\right)
$$

the potential closely resembles that of the previous section.

The main difference from the model of the previous section is the much larger number of degrees of freedom to be thermally averaged. We can thus expect the coefficients $\alpha_{i}$ to be larger than before. Further, the Higgs fields are not only self coupled but also coupled to the gauge bosons and the fermions, though, of the latter, only the top quark has an appreciable Yukawa coupling $\lambda_{t}$, and this, only to $\Phi_{1}$.

We will adjust the phases of the Higgs doublets so that the only fields in (3.3) which get a VEV are $\phi_{1}, \phi_{2}$ and $\psi_{2}$. Proceeding in the same fashion as before, we can compute three thermally averaged equations of motion:

$$
\begin{gathered}
\left\langle\frac{\partial V}{\partial \phi_{2}}\right\rangle=0=4 k_{2} \phi_{2}^{3}+\phi_{2}\left[\alpha_{2} T^{2}+2\left(k_{3}-k_{5}\right) \phi_{1}^{2}+4 k_{2} \psi_{2}^{2}-4 \mu_{2}^{2}\right]-2 \mu_{3}^{2} \phi_{1} \\
\left\langle\frac{\partial V}{\partial \psi_{2}}\right\rangle=0=4 k_{2} \psi_{2}^{3}+\psi_{2}\left[\alpha_{2} T^{2}+2\left(k_{3}+k_{5}\right) \phi_{1}^{2}+4 k_{2} \phi_{2}^{2}-4 \mu_{2}^{2}\right]-2 \mu_{4}^{2} \phi_{1} \\
\left\langle\frac{\partial V}{\partial \phi_{1}}\right\rangle=0=4 k_{1} \phi_{1}^{3}+\phi_{1}\left[\alpha_{1} T^{2}+2\left(k_{3}-k_{5}\right) \phi_{2}^{2}+2\left(k_{3}+k_{5}\right) \psi_{2}^{2}-4 \mu_{1}^{2}\right] \\
-2 \mu_{3}^{2} \phi_{2}-2 \mu_{4}^{2} \psi_{2} ;
\end{gathered}
$$


where

$$
\begin{aligned}
& \alpha_{1}=2 k_{1}+\frac{2}{3} k_{3}+\frac{1}{3} k_{4}+\frac{e^{2}\left(1+2 \cos ^{2} \theta_{W}\right)}{\sin ^{2} 2 \theta_{W}}+2 \lambda_{t}^{2} ; \\
& \alpha_{2}=2 k_{2}+\frac{2}{3} k_{3}+\frac{1}{3} k_{4}+\frac{e^{2}\left(1+2 \cos ^{2} \theta_{W}\right)}{\sin ^{2} 2 \theta_{W}},
\end{aligned}
$$

where $e$ and $\theta_{W}$ are the electromagnetic coupling and electroweak mixing angle respectively. For the moment, let us restrict our attention to the case when $k_{4}=k_{5}=0$ and $\mu_{3}=\mu_{4}=0$ so that there is no $\mathrm{CP}$ violation in the potential and the VEVs may both be taken to be real. With this simplification the equations (3.4) become the same as (2.3) except that, as expected, there are more contributions to $\alpha_{i}$. We will take $\lambda_{t}=0.5$ corresponding to a zero temperature top mass of $123 \mathrm{GeV}$.

The analysis of the transition is now almost identical to that described in the previous section. The eventual VEV is still $\phi_{1}=246 \mathrm{GeV}$, so if we choose as before $k_{1}=0.01$, $\mu_{1}=25 \mathrm{GeV}$, then again $m_{1}=70 \mathrm{GeV}$. Once more, choosing $k_{2}=0.8$, and this time $k_{3}=0.2$, we then immediately obtain from (3.5) $\alpha_{1}=0.987$ and $\alpha_{2}=2.07$. From (2.4) we then obtain $T_{c_{1}}=50 \mathrm{GeV}$. The choice $\mu_{2}=43 \mathrm{GeV}$ will then satisfy the remaining constraints. We find $m_{2}=130 \mathrm{GeV}$ to be the mass of the other neutral scalar as well as the charged scalars and the pseudoscalar. They are all equal in mass because of the residual symmetry in the Higgs sector when no component of $\Phi_{2}$ has a VEV. From (2.4) we find that the first transition occurs at $T_{c_{2}}=60 \mathrm{GeV}$ and from (2.8) we see that this is followed by a tunneling transition at $T_{t}=46 \mathrm{GeV}$, at which point, the VEV changes from $30 \mathrm{GeV}$ in the $\phi_{2}$ direction to $90 \mathrm{GeV}$ in the $\phi_{1}$ direction, as we can see by substitution in (2.5) and (2.6). Some of the details of this transition are displayed in fig. 1.

Reintroduction of certain of the missing terms causes no substantial changes. The main effect of a positive $k_{4}$ is to increase the charged scalar masses; these do not interest us at the moment, so we will leave this parameter vanishing. If we increase $k_{5}$ to a non-zero value, the equations (3.4) change, but not their solution. The extra scalar and pseudoscalar masses will also be split, but these are so large that we can allow $k_{5}$ to be almost as great as $k_{3}$ without running into any difficulties with experiment. The main effect of $k_{5}$ is to assure that the phases of $\Phi_{1}$ and $\Phi_{2}$ are aligned (that is, $\psi_{2}=0$ ) when they are both non-zero, so, even with these complications, we expect the phases of the two Higgs fields to be aligned in the transition region comprising the middle of the bubble wall. This term, however, will have very little effect on the regions near either of the two local minima, when one of the fields vanishes, and it is these regions that constitute the top and bottom of the wall. The effects of the $\mu_{3}^{2}$ and $\mu_{4}^{2}$ terms will be studied in the next section. 


\section{CP Violation and Weak Scale Baryogenesis}

We wish, now, to show that the transition which we have described may be useful in generating a baryon excess at the weak scale. The potential already has the necessary out of equilibrium phase transition, and electroweak interactions can violate baryon number, but we have not introduced CP violation. To achieve this, we will now reinstate $\mu_{3}$ and $\mu_{4}$; however, we will keep them sufficiently small (say $\mathcal{O}(1 \mathrm{GeV})$ ) that we only need consider perturbations about the positions of the minima computed in section 3 . We must return to (3.4) and calculate the corrected positions of the minima. Let's see what happens to the minimum in the $\phi_{1}$ direction. It will pick up small components in the $\phi_{2}$ and $\psi_{2}$ directions. The first two equations in (3.4) may be combined for non zero $\phi_{1}$ to yield:

$$
2 k_{5} \phi_{1} \phi_{2} \psi_{2}-\mu_{4}^{2} \phi_{2}+\mu_{3}^{2} \psi_{2}=0
$$

The first term here is second order in the small fields and so we obtain for the tangent of the CP violating phase: $\psi_{2} / \phi_{2} \approx \mu_{4}^{2} / \mu_{3}^{2}$. Similarly, the other minimum will pick up a small component in the $\phi_{1}$ direction. Either of the first two equations in (3.4) can be used to determine the magnitude of $\Phi_{2}^{0}$ by ignoring the small $\phi_{1}$ terms. With non-zero $\phi_{1}$, however, we are no longer free to choose $\psi_{2}$ to vanish, and hence the CP violating phase to be zero, and instead must determine it from equation (4.1). Once again, the first term is negligible because $\phi_{1}$ is perturbatively small and we obtain as before: $\psi_{2} / \phi_{2} \approx \mu_{4}^{2} / \mu_{3}^{2}$. The small component $\phi_{1}$ is then given approximately by the solution of the cubic derived from the third equation in (3.4), where, for $\phi_{2}$ and $\psi_{2}$ we use the values we have just calculated from the first pair of equations in (3.4).

The phase structure of a bubble wall can thus be estimated when we recall from the previous section that in the middle of the wall, where both $\phi_{1}$ and $\phi_{2}$ are large, the phase tends to be driven to zero so as to minimise the $k_{5}$ term in the potential. We can thus typically expect the variations in phase experienced by top quarks during reflection from the wall to be $\Delta \theta=\mathcal{O}\left(\arctan \mu_{4}^{2} / \mu_{3}^{2}\right)$. Naively, in the spirit of [7], we might expect the preferential reflection of hypercharge to be proportional to $\Delta \theta^{1}$, which may lead, ultimately,

1 We should note that, in the limit of the CP violating parameters tending to zero, the preferential reflection of top quarks with definite hypercharge will not be proportional to $\Delta \theta$. This is a reason for wishing to obtain a description of the transition without making these parameters too small. 
to a baryon asymmetry. Thus we see that the introduction of small $\mu_{3}$ and $\mu_{4}$ values may result in net baryon production at the phase transition wall.

For somewhat larger values of $\mu_{3}$ and $\mu_{4}$, we do not expect the essential features of the transition to be altered, but to obtain accurate results for the quantities involved, it becomes necessary to find the relevant solutions of (3.4) by numerical methods.

Recall from section 3, that at the jumping temperature, we have the following ratios of VEV to temperature : $v / T_{t}=0.67$ in the false vacuum; $v / T_{t}=2.0$ in the true vacuum. Baryon violation is suppressed in its rate by a factor of $\exp \left(-\frac{4 \pi}{g_{W}} v / T\right)$ [10, where $g_{W}$ is the $\mathrm{SU}(2)_{L}$ gauge coupling. When the VEV makes its jump during the second stage of the transition, the rate is thus reduced by a factor of approximately $4 \times 10^{-12}$, to a value which is slow enough to ensure that any baryon asymmetry created will not be subsequently dissipated. In false vacuum regions, in contrast, the baryon violation, whilst not completely unsuppressed, can still be rapid enough to convert hypercharge to baryon number before the bubble wall sweeps past. To generate the observed baryon asymmetry, therefore, we favour bubble walls with low velocities and a large $\mathrm{CP}$ violating parameter $\Delta \theta=\mathcal{O}\left(\arctan \mu_{4}^{2} / \mu_{3}^{2}\right)$.

\section{Conclusion}

In exploring the parameter space of two Higgs models, we have found that the symmetry breaking process may occur in two steps, the second of which produces many of the features of a strongly first order phase transition. We have shown that this may be important in certain weak scale baryogenesis scenarios, where it is crucial that the baryons do not disappear immediately subsequent to the transition, as happens if it is only weakly first order.

Calculation of higher order thermal loop graphs to obtain an improved effective potential will only yield small corrections since the interesting stage of the transition occurs well away from the origin in field space. It may be interesting, however, to work out more accurately, just how large a CP violating phase can be obtained by adjusting the various parameters. 


\section{References}

[1] A. Linde, Particle Physics and Inflationary Cosmology (Harwood Academic Publishers 1990) .

[2] G.W. Anderson and L.J. Hall, Phys. Rev. D45 (1992) 2685 ;

M. Dine et al., Phys. Lett. 283B (1992) 319 ;

M.E. Carrington, Phys. Rev. D45 (1992) 2933.

[3] C.G. Boyd, D.E. Brahm, S.D.H. Hsu, Work in Progress.

[4] A.I. Bocharev, S.V. Kuzmin and M.E. Shaposnikov, Phys. Lett. 244B (1990) 275 , Phys. Rev. D43 (1991) 369.

[5] N. Turok and J. Zadrozny, Nucl. Phys. 358B (1991) 471 ;

B. Kastening, R.D. Peccei and X. Zhang, Phys. Lett. 266B (1991) 413 ;

L. McLerran et al., Phys. Lett. 256B (1991) 451.

[6] N. Turok and J. Zadrozny, Nucl. Phys. 369B (1992) 729.

[7] A.G. Cohen, D.B. Kaplan and A.E. Nelson, Phys. Lett. 263B (1991) 86.

[8] A.E. Nelson, D.B. Kaplan, A.G. Cohen, Nucl. Phys. 373B (1992) 453.

[9] J.F. Gunion, H.E. Haber, G. Kane and S. Dawson, The Higgs Hunter's Guide (Addison-Wesley Publishing Company, 1990) .

[10] N.S. Manton, Phys. Rev. D21 (1980) 1591 ;

N.S. Manton, Phys. Rev. D28 (1983) 2019 ;

F.R. Klinkhamer and N.S. Manton, Phys. Rev. D30 (1984) 2212 ;

V.A. Kuzmin, V.A. Rubakov and M.E. Shaposhnikov, Phys. Lett. 155B (1985) 36 ;

P. Arnold and L. McLerran, Phys. Rev. D36 (1987) 581, Phys. Rev. D37 (1988) 1020 


\section{Figure Captions}

Fig. 1. Temperature development of the positions in field space of the minima of the effective potential and the vacuum expectation values of the scalars. 